\title{
Differentiation potential of human mesenchymal stem cells derived from adipose tissue and bone marrow to sinus node-like cells
}

\author{
JING YANG $^{1,2^{*}}$, TAO SONG ${ }^{1,2^{*}}$, PAN WU $^{1,2}$, YONGJUN CHEN $^{1,2}$, XINRONG FAN $^{1,2}$, \\ HUI CHEN ${ }^{1,2}$, JUN ZHANG ${ }^{1,2}$ and CONGXIN HUANG ${ }^{1,2}$ \\ ${ }^{1}$ Department of Cardiology, Renmin Hospital of Wuhan University; \\ ${ }^{2}$ Cardiovascular Research Institute, Wuhan University, Wuhan 430060, P.R. China
}

Received June 22, 2011; Accepted September 26, 2011

DOI: $10.3892 / \mathrm{mmr} .2011 .611$

\begin{abstract}
Adult mesenchymal stem cells (MSCs) hold great promise for the repair of heart defects. Both bone marrowderived mesenchymal stem cells (BMSCs) and adipose tissue-derived stem cells (ASCs) are multipotent and may be induced by 5 -azacytidine to differentiate into cardiomyocytes. However, the differentiation potential of human MSCs into sinus node-like cells has not been studied extensively. The aim of this study was to analyze the differences in proliferation and phenotype of ASCs and BMSCs from the same donors and to evaluate their capacity to differentiate into sinus node-like cells in vitro. Five passaged cells from bone marrow and adipose tissue were treated with $10 \mu \mathrm{M} 5$-azacytidine for $48 \mathrm{~h}$ and further cultured in complete medium for 4 weeks. A comparative study of cultured ASCs and BMSCs was carried out, and the morphological parameters, proliferative capacity, expression of surface markers and differentiation potential to sinus node-like cells were characterized. No morphologic differences were observed between ASCs and BMSCs. Flow cytometric analysis revealed that ASCs and BMSCs both expressed CD29, CD44, CD90 and CD105 and did not express CD34 and CD14, while CD49d, CD106 and CD34 were differentially expressed. Growth curves and doubling time determined with the Cell Counting Kit-8 (CCK-8) demonstrated that ASCs had a stronger proliferative ability than BMSCs. Histological immuofluorescence staining suggested that ASCs and BMSCs were capable of differentiating into
\end{abstract}

Correspondence to: Professor Congxin Huang, Cardiovascular Research Institute, Wuhan University, Division of Cardiology, Renmin Hospital of Wuhan University, 238 Jiefang Road, Wuchang, Wuhan 430060, P.R. China

E-mail: huangcongxing@yahoo.cn

*Contributed equally

Key words: mesenchymal stem cells, adipose tissue, bone marrow, cardiomyogenesis, $\mathrm{HCN}$ sinus node-like cells and that the positive expression ratios of cTNI were higher in ASCs compared to BMSCs at 4 weeks. Expression of the HCN2 and HCN4 genes was detected by reverse transcriptase polymerase chain reaction, and the results revealed that the expression of the $\mathrm{HCN}$ genes appeared earlier in ASC-derived sinus node-like cells. ASCs expressed HCN2 and HCN4 shortly after induction with 5-azacytidine for 2 weeks, although BMSCs expressed these genes after 4 weeks. The expression levels of HCN2 and HCN4 mRNA in ASC-derived cells were higher compared to those of BMSCs at 4 weeks. In conclusion, ASCs may be a better candidate as a novel source of cell therapy in sinus bradycardia disorders than BMSCs.

\section{Introduction}

Stem cell biology has gained increasing scientific and public interest in recent years. In particular, the use of stem cells in the treatment of heart disease has been strongly pursued (1). Mesenchymal stem cells (MSCs) represent a promising tool for new clinical concepts in supporting cellular therapy (2). A significant effort has gone into the use of adult MSCs for cardiac repair, including bone marrow (3), blood and cardiacderived cell populations. Bone marrow mesenchymal stem cells (BMSCs) were the first source reported to differentiate into cardiomyocytes in vitro (4). However, for clinical use, bone marrow may be detrimental due to the highly invasive donation procedure and the decline in MSC number and differentiation potential with increasing age. Recently, studies (5-7) have emerged on adipose-derived mesenchymal stem cells (ASCs) which also revealed a potential for cardiac lineage development in vitro (8) and may have application in cell-mediated in vivo therapy for the diseased heart (9-11). Adipose tissue (AT) is another alternative source that could be obtained by a less invasive method and in larger quantities compared to bone marrow (BM). These factors may make ASCs a viable clinical alternative to BMSCs. However, there is a need to more accurately compare these two populations. Therefore, in this study, we sought to compare ASCs and BMSCs in in vitro conditions with respect to their morphology, expansion characteristics, immunophenotype and cardiomyogenic 
differentiation capacity. Given donor variability and to make the comparison internally consistent, we compared ASCs and BMSCs obtained from the same donors.

\section{Materials and methods}

Harvest of adipose tissue and bone marrow. A bone marrow biopsy and a small piece of adipose tissue were obtained from 6 patients undergoing elective hip surgery after receiving informed consent. The patients included 3 males and 3 females ranging in age from 7 to 50 years (mean age of 37 ). All patients included in this study gave the pertinent informed consent and all experiments were approved by the Ethics Committee of Wuhan University.

Isolation and expansion of MSCs from adipose tissue. Human adipose tissue was washed with sterile phosphate-buffered saline (PBS) and digested by $1 \mathrm{mg} / \mathrm{ml}$ collagenase type I (Invitrogen) for $45 \mathrm{~min}$ at $37^{\circ} \mathrm{C}$ during permanent shaking. The cell suspension was centrifuged at $1000 \mathrm{x}$ g for $10 \mathrm{~min}$. The cells were washed and then cultivated in complete medium containing L-DMEM (Gibco, USA) supplemented with $10 \%$ fetal bovine serum (FBS; Hyclone), and $1 \%$ penicillin/ streptomycin (Invitrogen) in a $37^{\circ} \mathrm{C}$ incubator with a $5 \% \mathrm{CO}_{2}$ atmosphere. Cells were plated in medium at a density of $5 \times 10^{6}$ nucleated cells/100-mm tissue culture dish. The plating density used was based on previous studies (12). Following $72 \mathrm{~h}$ of culture, the medium was removed to discard unattached cells and then replaced with fresh medium. When the cells reached $80 \%$ confluence, they were detached from the culture flask using $0.25 \%$ trypsin containing $1 \mathrm{mM}$ EDTA (Sigma, USA) for further analysis.

Isolation and expansion of MSCs from bone marrow. BMSCs were obtained from 6 human bone marrow samples. Approximately $5 \mathrm{ml} \mathrm{BM}$ aspirate was collected in a syringe containing 10,000 IU heparin to prevent coagulation. BMSCs were separated from other bone marrow-residing cells by plastic adherence, and were then grown under tissue culture conditions. Briefly, the bone marrow aspirate $(5 \mathrm{ml})$ was combined with cells then resuspended and gently layered onto a Ficoll cushion (density, $1.073 \mathrm{~g} / \mathrm{ml}$; Invitrogen). The low-density BMSC-enriched mononuclear fraction was collected, washed with $10 \mathrm{ml}$ PBS and centrifuged to collect the cells. Cells were resuspended in complete culture medium, which consisted of Dulbecco's modified Eagle's medium with low glucose (Gibco), 10\% heat-inactivated FBS, penicillin (100 units/ml), and streptomycin $(100 \mu \mathrm{g} / \mathrm{ml})$ (Invitrogen). The cultures were maintained at $37^{\circ} \mathrm{C}$ in a humidified atmosphere containing $95 \%$ air and $5 \% \mathrm{CO}_{2}$. When 70 to $80 \%$ confluent, adherent cells were trypsinized $\left(0.25 \%\right.$ trypsin at $37^{\circ} \mathrm{C}$ for $\left.2 \mathrm{~min}\right)$, harvested and expanded in culture flasks.

Flow cytometric analysis. MSCs at passage 5 from adipose tissue and bone marrow $\left(1 \times 10^{6}\right)$ were suspended in $500 \mu 1$ PBS containing $20 \mathrm{ng} / \mathrm{ml}$ fluorescein isothiocyanate (FITC)coupled antibodies against CD29, CD44, CD73, CD90, CD105, CD49d, CD106 and CD34 (Biolegend). After incubation for $30 \mathrm{~min}$ at $4^{\circ} \mathrm{C}$, the cells were washed with PBS and resuspended in $1 \mathrm{ml}$ PBS for flow cytometry analysis. Cell fluorescence was evaluated by flow cytometry in a FACSCalibur instrument (Becton-Dickinson) and data were analyzed using CellQuest software (Becton-Dickinson). Flow cytometry applying fluoresceinated antibodies was used to determine surface molecules present in the expanded cell population, and both positive and negative surface molecules are listed.

Cytokinetics analysis by $C C K-8$. BMSCs and ASCs at the fifth passage were plated respectively in 96-well plates to develop growth curves, $10 \mu \mathrm{l}$ of Cell Counting Kit- 8 was added to each well and incubated for $3 \mathrm{~h}$ at $37^{\circ} \mathrm{C}$. The optical density values were determined in triplicate at a test wavelength of $450 \mathrm{~nm}$ and reference wavelength of $630 \mathrm{~nm}$. The doubling time was calculated during the logarithmic phase of the growth curve. The population doubling time was calculated using the formula (13):

$$
\mathrm{Td}=\mathrm{T} \times \frac{\log 2}{\log \frac{N t}{N 0}}
$$

where $\mathrm{T}$ is the time of the logarithmic phase of the growth curve, $N O$ is the number of cells after seeding and $N t$ is the number of cells at the end of the logarithmic growth phase.

Cardiomyogenic differentiation. ASCs and BMSCs at the fifth passage were treated in complete medium containing $10 \mu \mathrm{M}$ 5 -azacytidine (Sigma) for the induction of cardiomyogenic differentiation. Following incubation for $48 \mathrm{~h}$, the cells were washed twice with PBS (Gibco) and the medium was changed to complete medium without 5-azacytidine. The medium was changed every 3 days and the experiment was terminated 4 weeks after the drug treatment.

Immunostaining studies. To investigate the expression of cardiac-specific antigens in the MSCs-derived cardiomyocytes, differentiated cell cultures were washed and fixed with $4 \%$ paraformaldehyde in $0.1 \mathrm{M}$ phosphate buffer ( $\mathrm{pH} 7.4$ ). Following permeabilization with $0.1 \%$ Triton $\mathrm{X}-100$, the cells were incubated with the primary antibody (anti-cTnI, 1:200) (all from Santa Cruz Biotechnology) overnight at $4^{\circ} \mathrm{C}$. FITC-labeled goat anti-mouse IgG was used as the secondary antibody. DAPI was used for visualization of the nuclei. The cells were observed under a fluorescent microscope (Leica, Wetzlar, Germany).

Real-time PCR analyses of the HCN gene. Quantitative PCR was performed to study the expression of HCN2 and HCN4 mRNA. Total RNA was extracted from the MSC at 2 and 4 weeks following cardiomyogenic induction with the use of TRIzol reagent (Invitrogen), according to the manufacturer's instructions. Isolated RNA $(2 \mu \mathrm{g})$ was converted into cDNA using a high-capacity cDNA synthesis kit (Takara, Japan). The primers used for PCR amplification were synthesized by Sangon Gene Company (Shanghai, China) and are displayed in Table I. The PCR conditions were 1 cycle of $50^{\circ} \mathrm{C}$ for $2 \mathrm{~min}$ and $95^{\circ} \mathrm{C}$ for $10 \mathrm{~min}$, followed by 45 cycles at $95^{\circ} \mathrm{C}$ for $15 \mathrm{sec}$ and $58^{\circ} \mathrm{C}$ for $1 \mathrm{~min}$. Real-time PCR was performed with a ABI Prism 7500 sequence detection system (PE Applied Biosystems). The SYBR Green real-time PCR Master Mix kit 
Table I. Primers used for real-time RT-PCR.

\begin{tabular}{lll}
\hline Genes & \multicolumn{1}{c}{ Forward } & \multicolumn{1}{c}{ Reverse } \\
\hline HCN2 & CACCTACTGTCGCCTCTACTCA & GCACCATCTCACGGTCATATT \\
HCN4 & CGCATCCCGACTACTACGAAC & GGTCTCCTTGTTGCCCTTAGTG \\
GADPH & TCCTGCACCACCAACTGCTTAG & AGTGGCAGTGATGGCATGGACT \\
\hline
\end{tabular}
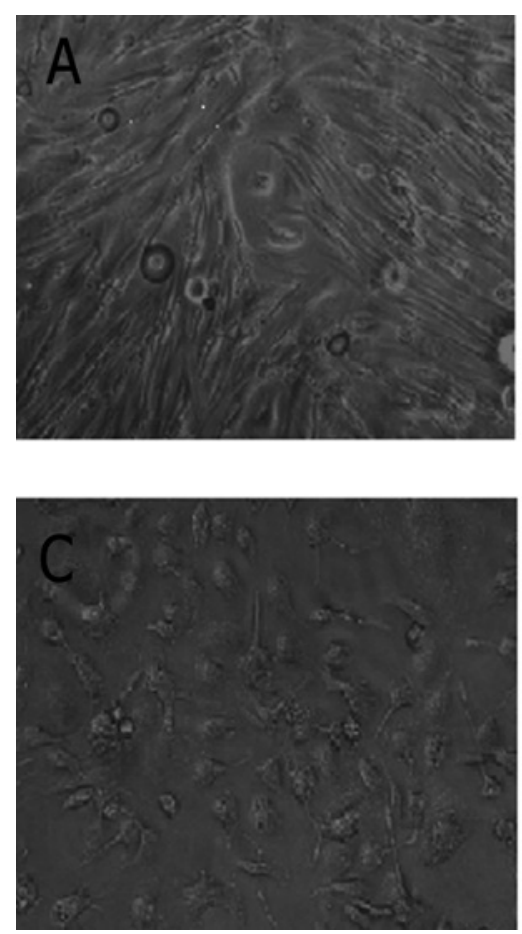
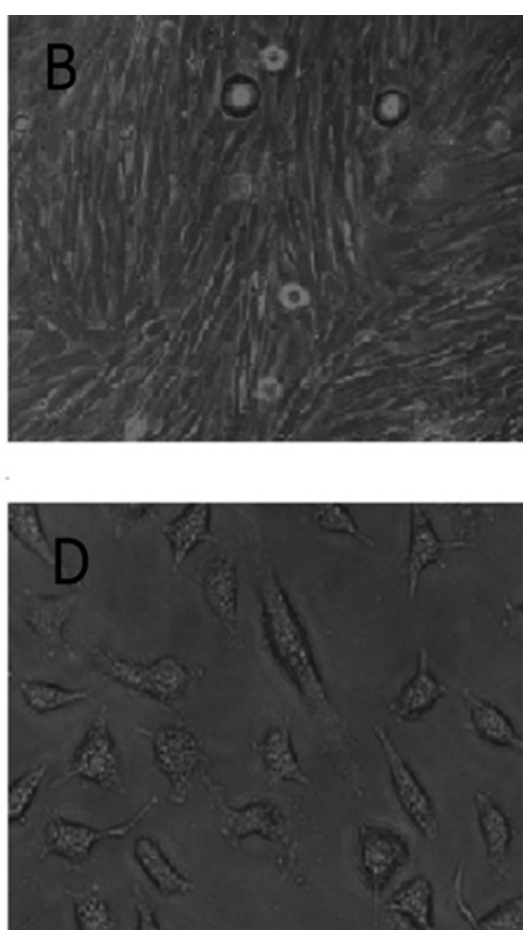

Figure 1. Phase-contrast images of mesenchymal stem cells (MSCs) before and after 5-azacytidine treatment. (A) Adipose tissue-derived stem cells (ASCs) revealed fibroblast-like morphology prior to 5-azacytidine treatment. (B) Bone marrow-derived mesenchymal stem cells (BMSCs) revealed fibroblast-like morphology prior to 5-azacytidine treatment. (C) ASC-derived cardiomyocytes formed a ball-like appearance following 5-azacytidine treatment. (D) BMSC-derived cardiomyocytes revealed a stick-like appearance following 5-azacytidine treatment.

(Takara) was utilized in subsequent PCR assays in accordance with the manufacturer's instructions. Semilog amplification curves were analyzed using the $2^{-\Delta \Delta C t}$ comparative quantification method and the expression of each gene was normalized to GAPDH.

Statistical analysis. Data are presented as the mean \pm standard deviation. A two-sided, non-paired $t$-test was used to analyze the flow cytometry and the cumulative doubling data. $\mathrm{P}<0.05$ was considered to denote statistically significant.

\section{Results}

Isolation of adherent cells from bone marrow and adipose tissue. Following an initial lag time of 2-3 days, cells entered a proliferative phase, reaching $80 \%$ confluence within 14 days. Adherent cells were observed following 4-7 days in culture. MSCs in culture appeared fibroblastic and homogeneous in size and morphology by the second passage. The morphology was similar in the ASCs and BMSCs (Fig. 1A and B). Following treatment with 5-azacytidine, the morphology of the cells gradually changed. Approximately $30 \%$ of the ASCs gradually increased in size and formed a ball-like appearance at 1 week, while BMSCs demonstrated multinucleation at 2 weeks and formed a stick-like appearance at 3-4 weeks (Fig. $1 \mathrm{C}$ and D).

Cell surface antigen expression. Cell surface antigen expression was evaluated by flow cytometry in at least 3 samples each from BMSCs and ASCs at passage 5. More than 80\% of the BMSCs and ASCs expressed the typical MSCs marker proteins (endoglin receptor CD105, extracellular matrix protein CD90, surface enzyme ecto-5'-nucleotidase CD73, b1-integrin CD29 and hyaluronate receptor CD44) although they lacked the hematopoietic marker CD34. However, statistical analyses comparing the MSCs sources regarding CD49d and CD106 revealed a significant difference between BMSCs and ASCs $(\mathrm{P}<0.01$; Table II).

Growth kinetics. At a plating density of $1 \times 10^{3}$ cells $/ \mathrm{cm}^{2}$, BMSCs at passage 5 reached $80 \%$ confluence by day 12 , while ASCs at the same passage reached this level by day 7 . The 

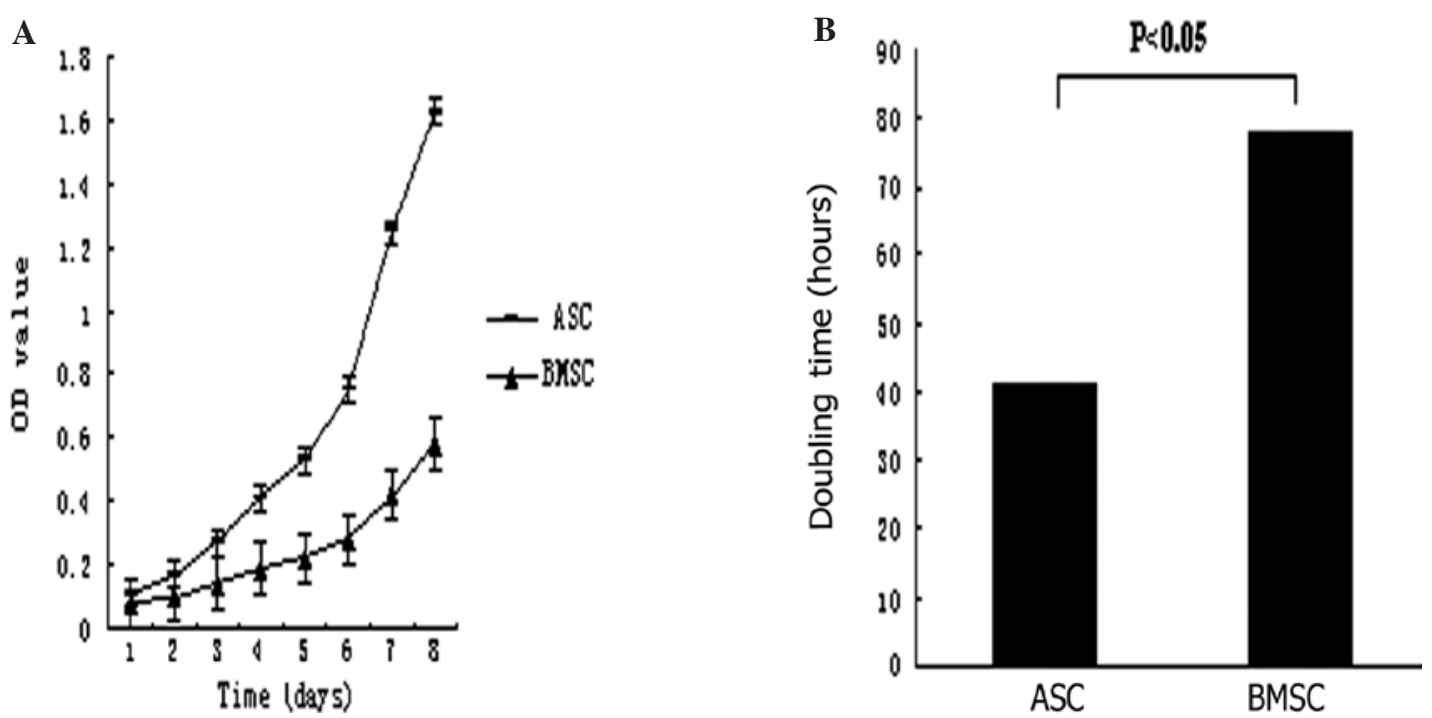

Figure 2. (A) Growth curve of bone marrow-derived mesenchymal stem cells (BMSCs) and adipose tissue-derived stem cells (ASCs) at passage 5. (B) Doubling time of ASCs and BMSCs.

Table II. Comparison of the surface protein expression of MSCs derived from BMSCs and ASCs as analyzed by flow cytometry.

\begin{tabular}{lrr}
\hline Antibodies & ASCs (\%) & BMSCs (\%) \\
\hline CD29 & $98.2 \pm 1.7$ & $98.7 \pm 2.3$ \\
CD44 & $96.4 \pm 1.2$ & $95.4 \pm 4.3$ \\
CD49d ${ }^{\text {a }}$ & $71.6 \pm 4.2$ & $18.1 \pm 2.7$ \\
CD73 & $91.0 \pm 2.5$ & $97.5 \pm 2.9$ \\
CD90 & $93.2 \pm 3.9$ & $93.1 \pm 3.5$ \\
CD105 & $82.3 \pm 5.1$ & $87.5 \pm 4.7$ \\
CD106 & $27.1 \pm 5.4$ & $70.3 \pm 9.5$ \\
CD34 & $1.6 \pm 0.7$ & $2.2 \pm 0.9$ \\
\hline
\end{tabular}

${ }^{\mathrm{a}} \mathrm{P}<0.01$. Each value is the mean percentage of at least three experiments \pm standard deviation.

mean population doubling times during the logarithmic phase of growth from days 3-7 were statistically significant $(78 \pm 16 \mathrm{~h}$ for BMSCs vs. $41 \pm 12$ h for ASCs; Fig. 2).

Immunostaining of differentiated cells for cardiac-specific antibodies. BMSCs and ASCs at passage 5 were induced with 5 -azacytidine for $48 \mathrm{~h}$. After cells had been cultured for 2 and 4 weeks, respectively, the expression of cTnI was examined by immunostaining. The results demonstrated that ASCs were specifically stained with anti-cardiac troponin I at 2 weeks, and BMSCs at 4 weeks. Fluorescence microscopic analysis revealed that the positive rates of ASCs and BMSCs differentiating into cardiomyocytes at 4 weeks were $34.5 \pm 3.2$ and $21.3 \pm 4.7 \%$, respectively. The percentage of ASCs that differentiated into cardiomyocytes following treatment with 5-azacytidine for 4 weeks was significantly higher compared to that of BMSCs $(\mathrm{P}<0.05$; Fig. 3A-C).
HCN gene expression in cardiomyocytes derived from MSCs. In order to detect the expression of $\mathrm{HCN}$ pacemaker channel genes in 5-azacytidine-treated MSCs, we isolated mRNA from BMSCs and ASCs after 2 and 4 weeks, respectively, of treatment with 5-azacytidine, and performed real-time PCR with primers specific for $\mathrm{HCN} 2$ and $\mathrm{HCN} 4$. In ASC-derived cells, the expression of HCN2 and HCN4 was detected after 2 weeks of treatment and this increased at 4 weeks. However, the HCN gene was detected until treated at 4 weeks in BMSCs, and RT-PCR demonstrated that the expression levels of HCN2 and HCN4 in ASCs induced for 4 weeks were stronger $(\mathrm{P}<0.05$; Fig. $4 \mathrm{~A}$ and $\mathrm{B})$.

\section{Discussion}

The possibility of using stem cell-based therapies has introduced a new therapeutic era for acute cardiovascular diseases $(7,10)$. MSCs from bone marrow and adipose tissue are attractive sources for cell-based therapy. Adipose tissue provides the opportunity to obtain stromal cells with multilineage potential from a much more readily available and abundant source $(14,15)$. However, the time required for isolation, expansion and differentiation of specific cell types could be a major handicap in the delivery of stem cells to acutely injured tissues or organs. This issue is of paramount significance in cardiovascular medicine. Lee et al (16) found that human adipose tissue-derived MSCs (AT-MSCs) were superior to bone marrow-derived stem cells in their proliferative ability. Factors such as donor age, culture conditions and culture media might influence the proliferative and differentiation capacity of MSCs. Therefore, a direct comparison of ASCs and BMSCs isolated from the same patients was performed. In this study, we isolated human MSCs from adipose tissue and bone marrow of the same donors to compare the morphology, proliferation ability, surface antigens and cardiomyogenic differentiation capacity of the two populations.

Consistent with other studies $(17,18)$, no difference in morphology was observed between ASCs and BMSCs. Prior to 


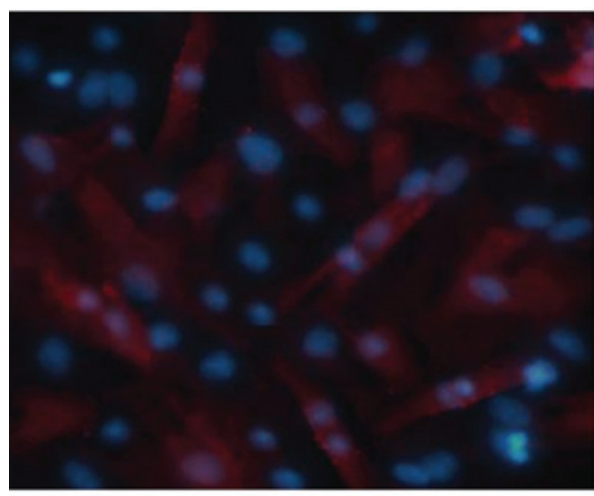

B

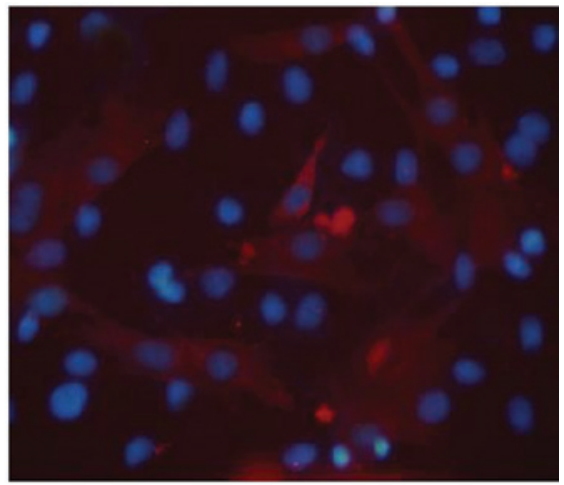

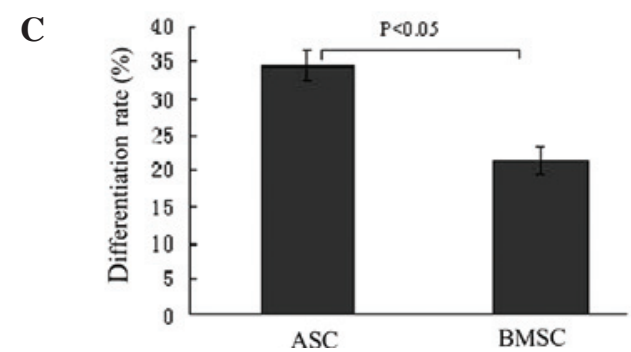

Figure 3. (A) Immunofluorescence staining of the adipose tissue-derived stem cells (ASCs) with anti-cTnI; cells were counterstained with DAPI to visualize the nucleus. (B) Immunofluorescence staining of the bone marrow-derived mesenchymal stem cells (BMSCs) with anti-cTnI; cell were counterstained with DAPI to visualize the nucleus. (C) Differentiation rate of ASCs and BMSCs.
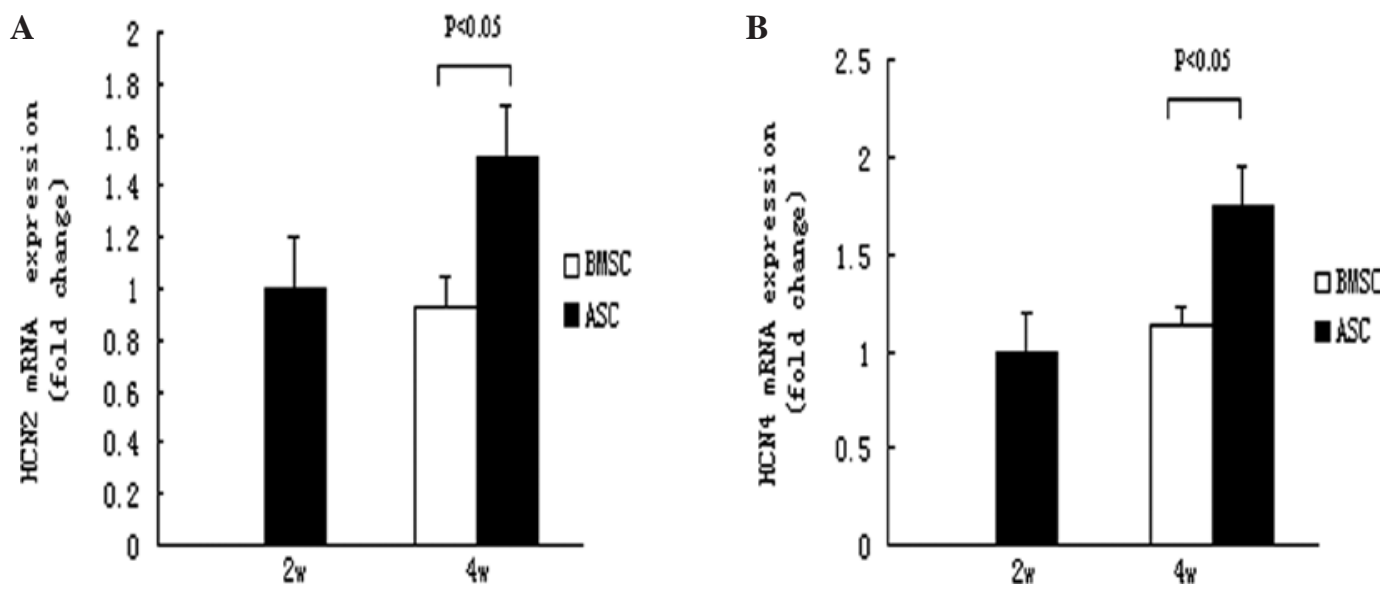

Figure 4. Real-time RT-PCR analysis of (A) HCN2 and (B) HCN4 in BMSC- and ASC-derived cardiomyocytes at 2 and 4 weeks, respectively, following treatment with 5-azacytidine.

treatment with 5-azacytidine, both ASCs and BMSCs stained positively for CD29, CD44 and CD105, although negatively for CD34 and CD45; however, the difference in surface marker expression appears to be with CD49d and CD106. The expression of CD49d, although not CD106, was observed in ASCs. Analysis of growth kinetics in vitro revealed that the doubling time of ASCs was much shorter compared to that of BMSCs. The results revealed that AT-MSCs are more proliferative than BMSCs. Thus, assuming clinical equivalence, the higher proliferative activity of ASCs would generate a clinically effective cell dose more rapidly than BMSCs.

Moreover, we investigated the ability of ASCs and BMSCs to differentiate in vitro into cardiomyocytes following treatment with 5-azacytidine. Tomita et al reported that the optimal concentration for cardiomyogenic differentiation is
$10 \mu \mathrm{M}$ (19). With the treatment of 5-azacytidine $(10 \mu \mathrm{M})$, the cardiomyocyte phenotype was identified by morphological observation, confirmed with immunofluorescence staining and gene expression of $\mathrm{HCN}$ We demonstrated that MSCs from both bone marrow and adipose tissue could be differentiated into cardiomyocyte-like cells following induction by 5 -azacytidine. We also observed that the time of cardiogenic differentiation in ASCs was shorter compared to that of BMSCs, and that the percentage of ASCs that differentiated into cardiomyocyte-like cells was significantly higher compared to that of BMSCs at the same time point following treatment with 5-azacytidine.

After being treated with 5-azacytidine, ASCs began to change their morphology and demonstrate multinucleation at 1 week, and formed a ball-like appearance thereafter, while 
BMSCs revealed multinucleation at 2 weeks and formed a stick-like appearance at 3-4 weeks. To characterize the differentiation of ASCs and BMSCs, we examined the expression of cTnI by immunostaining at weeks 2 and 4, respectively, following treatment with 5-azacytidine. The expression of cTnI was detected in the cultured ASCs at 2 weeks but in BMSCs at 4 weeks. Fluorescence microscopic analysis revealed that ASCs treated with 5-azacytidine had a higher expression of cardiac-specific marker troponin I compared with BMSCs at 4 weeks. ASCs differentiated into cardiac cells at a higher rate than BMSCs at 4 weeks.

Recent reports demonstrated that MSCs could differentiate into cardiac cells with pacemaking function in vitro. The hyperpolarization-activated, cyclic nucleotide-modulated (HCN) channel gene family is known to contribute significantly to the cardiac pacemaker current. In this study, we further investigated the mRNA expression of HCN2 and $\mathrm{HCN} 4$ in the cardiomyocytes derived from BMSCs and ASCs at 2 and 4 weeks, respectively. In adipose tissue-derived cardiomyocyte-like cells, there was clear mRNA expression for pacemaker channels HCN2 and HCN4 at 2 weeks. However, bone marrow-derived cells expressed HCN2 and HCN4 after 4 weeks. Real-time PCR demonstrated that the expression level of each gene in ASCs at 4 weeks was stronger compared to that in BMSCs.

In conclusion, MSCs from both adipose tissue and bone marrow could be induced by 5 -azacytidine to differentiate into sinus node-like cells (20). The present study demonstrated that the HCN family was expressed following differentiation (21). It has been suggested that patients with bradyarrhythmias could be treated by transplantation of regenerated sinus node-like cells from MSCs. ASCs might be a potential source of cell transplantation as an ideal seed cell for tissue engineering. The main benefit of ASCs is that they are easily harvested from patients by a simple, minimally invasive method and are also easily cultured. Moreover, cultured populations of ASCs may be expanded more rapidly and may have a superior potential for cardiomyogenesis compared with BMSCs, which suggests that ASCs could be a better candidate as a novel source of cell therapy in cardiovascular disease than BMSCs.

\section{Acknowledgements}

This study was supported by grants from the National Natural Science Foundation of China (no. 30700312).

\section{References}

1. Orlic D, Hill JM and Arai AE: Stem cells for myocardial regeneration. Circ Res 91: 1092-1102, 2002.

2. Pittenger MF, Mackay AM,Beck SC, et al: Multilineage potential of adult human mesenchymal stem cells. Science 284: 143-147, 1999.
3. Orlic D, Kajstura J, Chimenti S, et al: Bone marrow cells regenerate infarcted myocardium. Nature 410: 701-705, 2001.

4. Makino S, Fukuda K, Miyoshi S, et al: Cardiomyocytes can be generated from marrow stromal cells in vitro. J Clin Invest 103: 697-705, 1999.

5. Zuk PA, Zhu M, Ashjian P, et al: Human adipose tissue is a source of multipotent stem cells. Mol Biol Cell 13: 4279-4295, 2002.

6. Izadpanah R, Trygg C, Patel B, et al: Biologic properties of mesenchymal stem cells derived from bone marrow and adipose tissue. J Cell Biochem 99: 1285-1297, 2006.

7. Kondo K, Shintani S, Shibata R, et al: Implantation of adipose-derived regenerative cells enhances ischemia-induced angiogenesis. Arterioscler Thromb Vasc Biol 29: 61-66, 2009.

8. Planat-Benard V, Menard C, Andre M, et al: Spontaneous cardiomyocyte differentiation from adipose tissue stroma cells. Circ Res 94: 223-229, 2004.

9. Miranville A, Heeschen C, Sengenes C, Curat CA, Busse R and Bouloumie A: Improvement of postnatal neovascularization by human adipose tissue-derived stem cells. Circulation 110 : 349-355, 2004.

10. Heydarkhan-Hagvall S, Schenke-Layland K, Yang JQ, et al: Human adipose stem cells: a potential cell source for cardiovascular tissue engineering. Cells Tissues Organs 187: 263-274, 2008.

11. Okura H, Matsuyama A, Lee CM, et al: Cardiomyoblast-like cells differentiated from human adipose tissue-derived mesenchymal stem cells improve left ventricular dysfunction and survival in a rat myocardial infarction model. Tissue Eng Part C Methods 16: 417-425, 2010.

12. Zuk PA, Zhu M, Mizuno H, et al: Multilineage cells from human adipose tissue: implications for cell-based therapies. Tissue Eng 7: 211-228, 2001.

13. Zhu Y, Liu T, Song K, Fan X, Ma X and Cui Z: Adipose-derived stem cell: a better stem cell than BMSC. Cell Biochem Funct 26: 664-675, 2008

14. Yoshimura H, Muneta T, Nimura A, Yokoyama A, Koga H and Sekiya I: Comparison of rat mesenchymal stem cells derived from bone marrow, synovium, periosteum, adipose tissue, and muscle. Cell Tissue Res 327: 449-462, 2007.

15. Lindroos B, Boucher S, Chase L, et al: Serum-free, xeno-free culture media maintain the proliferation rate and multipotentiality of adipose stem cells in vitro. Cytotherapy 11: 958-972, 2009.

16. Lee RH, Kim B, Choi I, et al: Characterization and expression analysis of mesenchymal stem cells from human bone marrow and adipose tissue. Cell Physiol Biochem 14: 311-324, 2004.

17. De Ugarte DA, Morizono K, Elbarbary A, et al: Comparison of multi-lineage cells from human adipose tissue and bone marrow. Cells Tissues Organs 174: 101-109, 2003.

18. Arrigoni E, Lopa S, De Girolamo L, Stanco D and Brini AT: Isolation, characterization and osteogenic differentiation of adipose-derived stem cells: from small to large animal models. Cell Tissue Res 338: 401-411, 2009.

19. Tomita S, Li RK, Weisel RD, et al: Autologous transplantation of bone marrow cells improves damaged heart function. Circulation 100: II247-II256, 1999.

20. Astori G, Vignati F, Bardelli S, et al: 'In vitro' and multicolor phenotypic characterization of cell subpopulations identified in fresh human adipose tissue stromal vascular fraction and in the derived mesenchymal stem cells. J Transl Med 5: 55, 2007.

21. Tomita Y, Makino S, Hakuno D, et al: Application of mesenchymal stem cell-derived cardiomyocytes as bio-pacemakers: current status and problems to be solved. Med Biol Eng Comput 45: 209-220, 2007. 